\title{
Correction: Optimization of the electron transport layer in quantum dot light-emitting devices
}

Gary Zaiats, Shingo Ikeda and Prashant V. Kamat

Correction to: NPG Asia Materials 12:57

https://doi.org/10.1038/s41427-020-00237-0

Article published online 04 September 2020

Correction is being made to the Acknowledgement section

\section{Acknowledgements}

We thank the ND Energy Materials Characterization Facility (MCF) for the use of their XPS and thermal evaporator. We also thank Sachin Kinge for helpful discussions and Toyota Motors Europe for their support during the preliminary work. G.Z. acknowledges the postdoctoral support received from ND Energy, University of Notre Dame. S.I. thanks Osaka Municipal Technical Research Institute for supporting his stay at the Notre Dame Radiation Laboratory. P.V.K. acknowledges the support by the Division of Chemical Sciences, Geosciences, and Biosciences, Office of Basic Energy Sciences of the US Department of Energy through award DE-FC02-04ER15533. This is NDRL no. 5262 from the Notre Dame Radiation Laboratory.

The original article has been updated.

Published online: 21 December 2020 